\title{
Amputação primária no trauma: perfil de um hospital da região centro-oeste do Brasil
}

\author{
Primary amputation in trauma: a profile of hospital Center-west region of Brazil \\ Flavio Renato de Almeida Senefonte', Giuliano Rodrigo de Paiva Santa Rosa², Mauri Luiz Comparin³, \\ Marcos Rogério Covre 4 , Mauricio de Barros Jafar ${ }^{5}$, Fábio Augusto Moron de Andrade, \\ Guilherme Maldonado Filho7, Ed Nogueira Neto ${ }^{8}$
}

\section{Resumo}

Contexto: Vivemos num período de epidemia do trauma. A amputação de indicação traumática incide em uma população jovem e economicamente ativa com repercussão onerosa no âmbito socioeconômico, tornando-se um problema de saúde pública.

Objetivo: Conhecer a casuística de amputações traumáticas realizadas na Santa Casa de Campo Grande-MS, entre 2005 e 2008.

Métodos: Estudo de prevalência, descritivo, longitudinal e retrospectivo. Amostragem de conveniência, realizada com revisão sistemática de prontuários de pacientes submetidos a amputações de membros inferiores e/ou superiores cuja indicação foi trauma incompatível com reconstrução. Foram excluídos os pacientes que já chegaram amputados no pronto-socorro. Avaliaram-se nível de amputação, faixa etária, sexo e escala do sistema MESS para indicação de amputação traumática. Utilizaram-se o teste quiquadrado e o teste exato de Fisher, considerando um intervalo de confiança de $95 \%$.

Resultados: Foram realizadas 108 amputações no período, na faixa etária de dois anos a 78 anos, com média de 36,7 \pm 12 anos e mediana de 35 anos. Houve predomínio do sexo masculino em $72 \%$ da casuística. O nível de amputação mais executado foi de amputações menores (pododáctilos e quirodáctilos). A causa mais frequente foi lesão decorrente de acidente de trânsito.

Conclusões: As amputações traumáticas atingiram uma população jovem e produtiva, conforme corroborado pela literatura, com predomínio de acidentes de trânsito com lesões ortopédicas e neurológicas associadas.

Palavras-chave: amputação; desarticulação; trauma.

\footnotetext{
Abstract

Background: We live in a period of epidemic of trauma. Amputation due to trauma affects a young and economically active population, with costly socioeconomic consequences, becoming a public health problem.

Objective: To investigate the series of amputations performed at Santa Casa de Campo Grande Hospital between 2005 and 2008.

Methods: Prevalence, descriptive, longitudinal and retrospective study. Convenience sampling was used to conduct a systematic review of medical records of patients undergoing lower and/or upper limb amputations whose diagnosis was trauma incompatible with reconstruction. Patients who arrived at the emergency department with amputated limbs were excluded from the study. We assessed the level of amputation, age, sex, and MESS score for amputation after trauma. The chi-square test and Fisher's exact test were used considering a 95\% confidence interval.

Results: One hundred and eight amputations were performed in the period. Patients' two to 78 years; mean age was $36.7 \pm 12$ years, and median age was 35 years. Most patients were males (72\%). The most frequent level of amputation was minor amputations (toes and fingers). The most common cause of amputation was injury resulting from traffic accidents.

Conclusions: In agreement with the literature, trauma amputations affect a young and productive population, with prevalence of traffic accidents with associated orthopedic and neurological injuries.

Keywords: amputation; disarticulation; trauma.

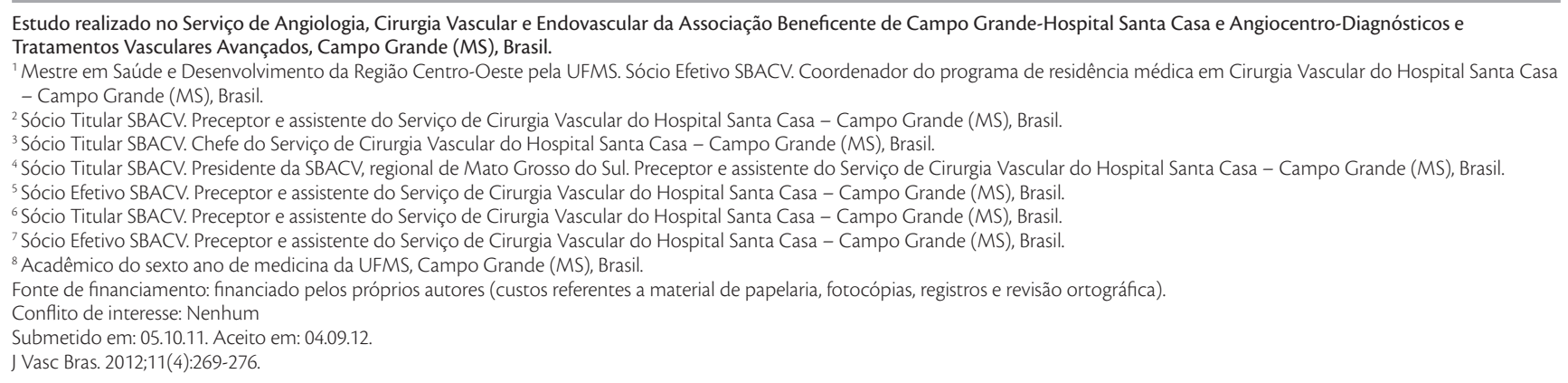




\section{Introdução}

A amputação de um membro é um dos recursos terapêuticos mais antigos da medicina ${ }^{1}$. No caso de sua indicação no trauma de membros por lesões graves de nervos, artérias, partes moles e ossos, esperava-se que diminuiria sua incidência com o fim das grandes guerras². Entretanto, houve a inversão do trauma de origem de conflito militar pelo trauma de origem civil, especialmente em virtude dos acidentes de trânsito, seguidos pela violência urbana $^{1-3}$.

O trauma é a grande pandemia da vida moderna e, ao que parece, veio para ficar. Cada vez mais o número de vítimas sobe e, estas, com uma característica preocupante, que é envolver uma faixa etária que compreende adultos jovens e economicamente ativos ${ }^{2}$. Suas consequências seguem nas mesmas proporções com importante impacto social quando fica sequela, como a amputação de um membro em virtude das lesões sofridas ${ }^{2}$.

A melhora econômica da população de países em desenvolvimento, como o Brasil, e o incremento da produção da indústria automobilística associada a um número cada vez maior de veículos em circulação acompanham-se de um absurdo aumento de acidentes, que, frequentemente, envolvem traumas graves dos membros inferiores.

Em contrapartida, observa-se pouca mobilidade no sentido de promoção de saúde para coibir novos acidentes. Ao deixar a prevenção apenas sob responsabilidade da consciência do cidadão sobre legislação de trânsito e penalidades previstas, acompanhada de escassa fiscalização, o trânsito se torna ambiente mais hostil. $\mathrm{O}$ círculo vicioso continua nos hospitais e serviços de atendimento de urgências que geram mais ônus aos escassos orçamentos destinados à saúde.

Faltam políticas preventivas mais competentes que impliquem maior comoção social sobre a realidade disfarçada ao grande público, exceto aos prestadores de serviços de saúde e suas vítimas sequeladas.

Diante desta triste realidade, apesar do avanço da medicina, ainda existe uma incidência elevada de amputações primárias no trauma ${ }^{2}$. Cabe a nós médicos zelar pela sua indicação ao estritamente necessário, tarefa também difícil de ser tomada, uma vez que envolvem múltiplos aspectos. Para lidar com o manejo destes traumas severos, pode ser necessária a adoção de alguns critérios como o Mangled Extremity Severety Score (MESS), ${ }^{4,5}$, uma escala de gravidade das lesões para apoiar a decisão terapêutica.

Visando melhor entender a ocorrência desses procedimentos, faz-se necessário empreender estudos epidemiológicos dos vários serviços disponíveis no Brasil e no mundo, a fim de se levantarem dados que permitam subsidiar um planejamento profilático voltado a reduzir ao estritamente necessário a indicação de amputação de um membro, procedimento cujo impacto econômico não é menor que o psicossocial. Devido à escassez de trabalhos no gênero, principalmente no Brasil, o presente estudo buscou alimentar a base de pesquisa científica e manter ativa a discussão sobre o problema para conhecer a casuística das amputações primárias decorrentes de trauma das extremidades. Assim, o objetivo principal deste artigo foi levantar um perfil epidemiológico da amputação de indicação primária, ou seja, sem tentativa de salvamento de membro, decorrente do trauma vascular de membros inferiores e superiores, atendidos no maior hospital da região e único a atender alta complexidade numa abrangência de dois milhões de habitantes no período de janeiro de 2005 a dezembro de 2008.

\section{Métodos}

Este estudo predominantemente descritivo, longitudinal e retrospectivo baseou-se na revisão sistemática de prontuários de pacientes submetidos a amputações primárias dos membros inferiores e superiores no período de janeiro de 2005 a dezembro de 2008 , com busca ativa de dados como idade, sexo, história do trauma e propedêutica realizada. Pesquisaram-se mecanismo do trauma, traumas associados, métodos diagnósticos empregados, tempo decorrente entre o primeiro atendimento hospitalar até o cirurgião vascular e porque não houve tentativa para salvamento de membros e sua consequente indicação de amputação primária. Neste sentido, buscou-se correlacionar o uso de uma escala de decisão sobre indicar ou não a amputação primária ou realizar uma tentativa de salvamento de membro. Entre as diversas que a literatura descreve, foi optado nesta pesquisa pelo uso da escala de MESS (ver discussão), escala empregada tanto pelo serviço de ortopedia quanto pelo de cirurgia vascular do hospital em questão.

Foram excluídos os pacientes que já chegaram amputados no pronto-socorro.

A análise dos dados considerou a observação de valores mínimos e máximos, calculando-se médias e desvios padrão. Para a análise de significância sobre a distribuição de frequências entre sexo e faixa etária utilizou-se o teste quiquadrado. $\mathrm{O}$ nível de significância pré-definido para rejeitar a hipótese de nulidade, quando aplicável, foi de 5\% $(\mathrm{p}<0,05)$. 


\section{Resultados}

No período de pesquisa foram realizados 122 procedimentos em 108 pacientes com idades que variaram de dois anos a 78 anos, com média de $36,7 \pm 12$ anos e moda e mediana de 35 anos. A estratificação dos pacientes por idade, conforme os critérios utilizados no MESS, revelou que a maioria tinha idade abaixo dos 30 anos, como se observa na Tabela 1 . O sexo masculino predominou com $72 \%$ contra $28 \%$ do sexo feminino (razão de 2,6:1) (Figura 1). Não houve associação significativa entre sexo e faixa etária (Tabela 1 ).

A distribuição do número de amputações primárias pode ser observada na Figura 2.

O processo de decisão pela amputação traumática foi utilizado pelo cirurgião vascular, associado muitas vezes ao ortopedista e compreenderam os seguintes aspectos, baseados na escala de MESS (ver discussão):

- Mecanismo do trauma;

- Tempo de isquemia e pesquisa de sinais de isquemia irreversível;

- Comorbidades com risco elevado de amputação secundária (idade avançada, diabetes, etc.);

- Condições de recuperação funcional (qualificação das lesões ósseas, musculares, dos nervos e dos vasos sanguíneos);

Tabela 1. Frequência dos casos de amputação primária, por faixa etária e sexo, do Hospital Santa Casa de Campo Grande-MS, 2005-2008.

\begin{tabular}{|c|c|c|c|c|c|c|}
\hline \multirow{3}{*}{ Faixa etária } & \multicolumn{4}{|c|}{ Sexo } & \multirow{2}{*}{\multicolumn{2}{|c|}{ Total }} \\
\hline & \multicolumn{2}{|c|}{ Masculino } & \multicolumn{2}{|c|}{ Feminino } & & \\
\hline & $\mathrm{n}$ & $\%$ & $\mathrm{n}$ & $\%$ & $\mathrm{n}$ & $\%$ \\
\hline Até 30 anos & 38 & 48,7 & 14 & 46,7 & 52 & 48,1 \\
\hline 31 a 50 anos & 27 & 34,6 & 10 & 33,3 & 37 & 34,3 \\
\hline Acima de 50 anos & 13 & 16,7 & 6 & 20 & 19 & 17,6 \\
\hline Total & 78 & 72 & 30 & 28 & 108 & 100 \\
\hline
\end{tabular}

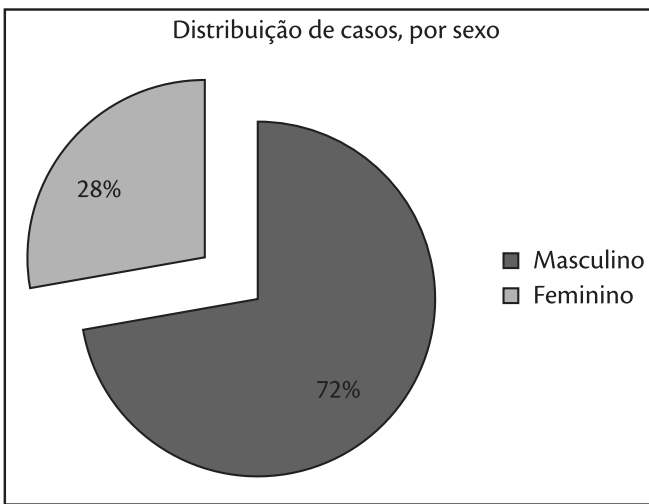

Figura 1. Distribuição dos casos de amputação, por sexo, no Hospital Santa Casa de Campo Grande-MS, 2005-2008.
- Possibilidade e disponibilidade de reparo vascular: calibres, sítio de doação do enxerto, cobertura adequada da derivação/interposição por pele e ou partes moles, qualidade do enxerto quando necessário (extensão, parede preservada e calibre);

- Grau de contaminação e risco de infecção;

- Estado hemodinâmico e cardiopulmonar que exigem salvamento da vida em detrimento do membro.

Quase a totalidade destes itens esteve presente ou foi neles baseada a decisão pela amputação primária nesta pesquisa.

Em relação à etiologia dos traumas de extremidades que foram submetidos a amputações primárias, observase a participação quase esmagadora dos acidentes automobilísticos seguidos por queimaduras, choques elétricos e traumas iatrogênicos (Figura 3).

Entre os casos de amputação por trauma, destacamos dois casos decorrentes de lesão por choque elétrico e um caso por lesão extensa em pé por queimadura.

Os níveis de amputação foram estratificados em maiores (membros superiores, transfemorais, transtibiais e desarticulações de quadril, joelho e tornozelo/pé) e menores (antepé, pododáctilos, quirodáctilos, etc.). Para melhor análise, os procedimentos também foram subdivididos em

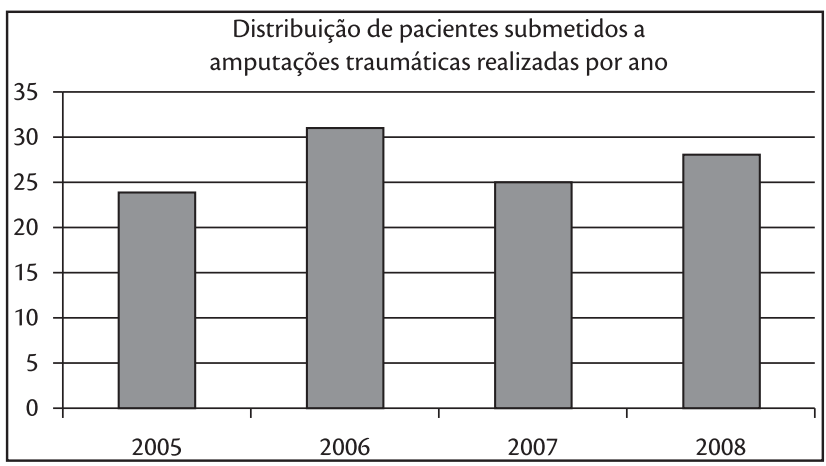

Figura 2. Distribuição das amputações traumáticas realizadas, por ano entre 2005 e 2008 no Hospital Santa Casa de Campo Grande-MS.

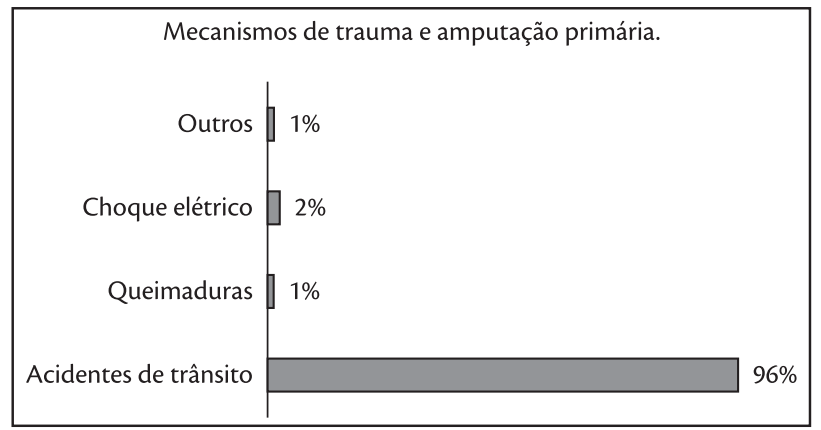

Figura 3. Mecanismos de trauma como etiologia das amputações primárias no Hospital Santa Casa de Campo Grande-MS, 2005 a 2008. 
amputações de membro superior $(1,85 \%$ de prevalência, exceto quirodáctilos), desarticulações de quadril (0,09\%), amputações transfemorais (13,8\%), desarticulações de joelho $(0,09 \%)$, amputações transtibiais $(21,3 \%)$ e operações no pé como desarticulação de tornozelo/pé, amputação parcial de pé $(26,8 \%)$ e amputação/desarticulação de hálux e/ou de pododáctilos e quirodáctilos, perfazendo $34,2 \%$ dos procedimentos (Figura 4).

Dezessete pacientes foram submetidos a mais de uma amputação (em outro nível e/ou outro membro) durante a mesma internação, seja por comprometimento infeccioso do membro residual ou por isquemia secundária a trombose proximal.

O membro mutilado, objeto da presente amostra, teve a sua relação observada segundo dados como traumas associados no membro (vascular, ortopédico, neurológico e suas combinações), fatores dos doentes (idade, comorbidades clínicas e tabagismo) e do trauma (mecanismo da lesão, lesões específicas, choque, hipotermia, tempo decorrido do trauma e atendimento) que acarretariam pior prognóstico na tentativa de salvamento do membro.

Entre os traumas associados, a lesão ortopédica predominou com $93,5 \%$ dos casos, com as fraturas/luxações expostas Gustilo IIIC, na sua esmagadora maioria. Traumas múltiplos envolvendo crânio, tórax e abdome estiveram presentes em $1,85 \%$ dos casos.

Quanto aos fatores associados aos doentes, a idade acima de 50 anos teve $17,6 \%$ de frequência e comorbidades clínicas como diabetes, insuficiência cardíaca, pneumopatias, doença vascular obstrutiva periférica, que não puderam ser totalmente analisadas devido a pequeno registro destes dados nos prontuários.

Já fatores relacionados ao trauma tiveram maior registro nos prontuários pesquisados. Entre eles, o choque hipovolêmico teve uma frequência de $9,3 \%$ da presente amostra. A hipotermia não teve registro encontrado e o

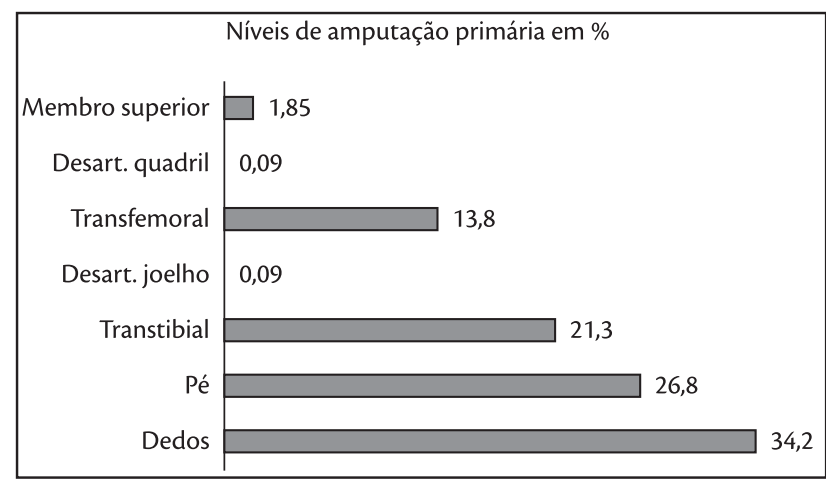

Figura 4. Frequência dos níveis de amputação primária realizada no Hospital Santa Casa de Campo Grande-MS entre 2005 e 2008. tempo decorrido do trauma ao atendimento especializado foi em média, de $5,3 \pm 1,8$ horas.

O período médio de internação foi de $23,7 \pm 7,8$ dias, com mediana de 29 dias e moda de 28 dias. Em relação à morbimortalidade, houve um óbito (paciente politraumatizado, submetido a amputação de antebraço esquerdo e desarticulação de quadril esquerdo, vítima de capotamento de caminhão) e dois casos de sepse.

Segundo os critérios da escala de pontos de MESS (ver adiante na discussão), que considera como pontos de corte as somas iguais ou maiores do que sete, nos dados disponíveis da presente amostra (68), observamos 13 casos em que a soma dos dados foi de cinco pontos, porém, nestes casos, foi considerado trauma com perda extensa de partes moles e laceração das artérias distais que impossibilitavam a reconstrução vascular. Nestes casos, a extremidade comprometida era perna distal ou pé.

A decisão de amputar foi tomada em conjunto com o ortopedista em $96,7 \%$ dos casos. Outras indicações encontradasforam a falta de um substituto vascular adequado (em comprimento ou sítio de doação comprometido) em dois casos, lesões com desenluvamento de pele, grande perda de substância para cobrir uma derivação/interposição vascular, associadas a fraturas cominutivas com mau prognóstico ortopédico e amplamente contaminadas, em $47 \%$ dos casos. O tempo prolongado de isquemia até avaliação de um especialista também teve papel importante, principalmente nos politraumatizados, com traumas vasculares contusos que foram submetidos primeiramente a laparotomias, toracotomias e/ou neurocirurgias até ser avaliada a necessidade da intervenção vascular. Neste momento, o membro, ou mesmo o pé ou a mão, apresentava sinais de rigidez, acompanhado de frialdade e paralisia, tornando inviável uma intervenção. Este cenário foi descrito em cerca de 30\% dos casos. Em 11\% da amostra, foi colocada pelo ortopedista a indicação por lesão dos nervos tibiais, sendo que, em dois casos, poderia ter sido tentada revascularização pelo cirurgião vascular.

\section{Discussão}

O mecanismo do trauma na síndrome do membro mutilado é, na sua maioria, contuso, com lesões associadas que determinam a impossibilidade de revascularização e, por isso, indica-se a amputação traumática. Os traumas penetrantes que envolvem grande perda tecidual como os oriundos de projéteis de alta velocidade também são relatados na literatura, entretanto, não tivemos nenhum caso. 
A amputação de membros é o recurso final ante uma doença crônico-degenerativa que evolui com isquemia e morte celular de uma extremidade ou ante um processo de destruição maciça que impede o restabelecimento circulatório. O propósito dessa intervenção é o de evitar a isquemia e suas consequências, que ocorrem em casos de trauma ${ }^{1,6,7}$.

No primeiro caso, a adoção de meios de prevenção e tratamento nas fases iniciais contribui para diminuir a utilização desses procedimentos ${ }^{8}$, cujo número total, porém, tem crescido em razão da crescente longevidade humana ${ }^{9}$ e ao aumento na prevalência de diabetes mellitus, obesidade, síndrome metabólica e dislipidemia, agravos que estão associados com menor qualidade de vida (estresse, sedentarismo, má educação alimentar e tabagismo) e com maior risco de doenças crônico-degenerativas, como nefropatias, hipertensão arterial sistêmica, pneumopatias e doenças cardiovasculares ${ }^{7,10-14}$.

A incidência mundial de amputação de membros é estimada em mais de um milhão ao $\mathrm{ano}^{7}$, cuja principal indicação decorre de complicações de diabetes e doença aterosclerótica $^{15}$, embora ainda sejam poucos os estudos voltados a estabelecer a epidemiologia dessa população ${ }^{6,16-19}$.

O crescimento das publicações sobre o tema ampliará o conhecimento do perfil desses pacientes, permitindo melhores perspectivas para a efetividade da prevenção, objeto da saúde pública ${ }^{20}$.

$\mathrm{O}$ presente estudo, por seu caráter descritivo e retrospectivo, visou levantar a prevalência das amputações realizadas no hospital pesquisado, acompanhado de seu perfil epidemiológico e associando índices de gravidade de lesão que reforçavam ou não a indicação de amputação primária.

As principais limitações do presente estudo foram as dificuldades para a coleta de dados em alguns prontuários, fato apontado por Almeida Filho e Rouquayrol ${ }^{21}$ como inerente aos estudos retrospectivos, os quais, por outro lado, têm a vantagem de ser menos dispendiosos e de execução mais rápida ${ }^{7}$.

O membro mutilado, objeto da presente amostra teve a sua relação observada segundo dados como traumas associados no membro (vascular, ortopédico, neurológico e suas combinações), fatores dos doentes (idade, comorbidades clínicas e tabagismo) e do trauma (mecanismo da lesão, lesões específicas, choque, hipotermia, tempo decorrido do trauma e atendimento) que ajudam a determinar o prognóstico na tentativa de salvamento do membro ${ }^{22}$. Os pacientes submetidos a uma tentativa de salvamento de membro que posteriormente foram submetidos à amputação, não foram objeto deste estudo, sendo excluídos da amostragem.

Por falta de registros, não foi possível determinar completamente o número de diabéticos, pneumopatas, cardiopatas, pacientes com osteoporose, pacientes com insuficiência vascular periférica e tabagistas ${ }^{2,4}$.

A importância epidemiológica de se conhecerem esses fatores reside no papel de que a sua presença no trauma pode contribuir para piorar o prognóstico de uma tentativa de salvamento de membro conforme suas relações com perfusão, cicatrização e resistência orgânica para recuperação e ou enfrentamento de situações adversas como infecção ${ }^{4}$.

Pacientes com menos de 50 anos predominaram na amostra, o que corresponde a um período da vida economicamente ativo ${ }^{23}$. O predomínio do sexo masculino, com uma relação de 2,6:1, também foi semelhante a dados da literatura ${ }^{7,24}$.

O teste quiquadrado revelou não haver associação significativa entre sexo e faixa etária $(\mathrm{p}=0,7386$, Tabela 1$)$, mas, em pacientes do sexo feminino, houve um menor número de amputações, resultado este semelhante aos disponíveis na literatura ${ }^{7,25}$.

A amputação decorrente de trauma, outrora de menor frequência, exerce grande impacto socioeconômico, por afetar uma faixa etária jovem, predominantemente do sexo masculino, no período mais produtivo da vida ${ }^{2,4,7,26}$.

Na presente amostra, o grande número de amputações e ou desarticulações de dedos foi mais prevalente em crianças, em concordância com a literatura que afirma que, nessa fase da vida, em especial a faixa etária pré-escolar e escolar, as amputações primárias por traumas decorrem de traumatismos em artelhos ao invés de traumas maiores de membros $2,7,27$.

Amputação primária é definida como a amputação na admissão, sem tentativa de revascularização. A sua decisão é extremamente complexa ${ }^{4}$.

Alguns fatores devem ser considerados para ajudar nesta decisão, relacionados ao doente, ao local do trauma e à equipe de atendimento ao traumatizado ${ }^{4}$.

A decisão de uma amputação primária no trauma nunca é fácil e deve ser tomada com muito bom senso, principalmente quando a tentativa de se preservar a extremidade acarretar risco de vida.

Para tanto, diversas escalas foram criadas com a intenção de facilitar a sua indicação ${ }^{4}$. Estas escalas graduam por pontos variáveis envolvidos no trauma e, se a soma destes pontos ultrapassar o corte, a amputação primária deve ser fortemente considerada. Entretanto, nenhuma das várias escalas existentes é considerada ideal. 
A escala de graduação mais usada é a MESS (Quadro 1), mas também a literatura propõe as seguintes escalas: Mangled Extremity Syndrome Index (MESI), Predictive Salvage Index (PSI), Hannover Fracture Scale (HFS), Nerve injury, ischemia, soft tissue injury, skeletal injury, shok and age of pacient Score (NISSSA), Limb Salvage Index (LSI) ${ }^{4}$.

Estas escalas, analisadas por Razuk et al. ${ }^{28}$ colaboram na decisão do procedimento a se realizar, contudo, sua maioria tem falhas metodológicas que não permitiram sua reprodução em estudos independentes.

$\mathrm{Na}$ presente amostra, não se objetivou observar o uso da escala de MESS e inferir sobre os casos de amputação primária nos membros mutilados, pois o estudo é retrospectivo. Por isso, analisou-se, nos casos que tiveram registros suficientes para preencher os dados para estes critérios, se correspondiam às pontuações de corte que sugerem uma forte indicação ao procedimento avaliado.

O MESS foi escolhido, porque, entre a literatura pesquisada, é o mais amplamente utilizado, possui poucas variáveis e parece ser fácil aplicá-lo. Os pontos de corte no MESS são maiores ou iguais a sete ${ }^{4}$.

No presente estudo, em apenas 68 pacientes houve dados suficientes para preencher os critérios de MESS e, destes, em 13 a soma foi inferior a sete pontos. No entanto, nestes casos, a amputação foi indicada devido

Quadro 1. Critérios utilizados no Mangled Extremity Severety Score.

\begin{tabular}{|c|c|}
\hline Lesões ósseas e de partes moles & Escore \\
\hline $\begin{array}{l}\text { Baixa energia: PAF, fraturas simples, } \\
\text { lesões incisas. }\end{array}$ & 1. \\
\hline $\begin{array}{l}\text { Média energia: fraturas expostas, } \\
\text { luxações. }\end{array}$ & 2. \\
\hline $\begin{array}{l}\text { Alta energia: esmagamentos, PAF de alta } \\
\text { velocidade. }\end{array}$ & 3. \\
\hline $\begin{array}{l}\text { Presença de contaminação grosseira e } \\
\text { avulsão de partes moles. }\end{array}$ & 4. \\
\hline Isquemia do membro & $\begin{array}{c}\text { Acima de } 6 \mathrm{~h} \text {, dobrar os } \\
\text { pontos. }\end{array}$ \\
\hline $\begin{array}{l}\text { Pulso diminuído ou ausente, mas com } \\
\text { boa perfusão. }\end{array}$ & 1. \\
\hline $\begin{array}{l}\text { Ausência de pulsos, parestesias, diminu- } \\
\text { ição do preenchimento capilar. }\end{array}$ & 2. \\
\hline Ausência de pulso, frio e paralisia. & 3. \\
\hline \multicolumn{2}{|l|}{ Choque } \\
\hline PAS $>90 \mathrm{mmHg}$. & 0. \\
\hline Hipotensão transitória & 1. \\
\hline Hipotensão persistente & 2. \\
\hline \multicolumn{2}{|l|}{ Idade } \\
\hline Abaixo de 30 anos & 0. \\
\hline Entre $30-50$ anos & 1. \\
\hline Acima de 50 anos & 2. \\
\hline
\end{tabular}

PAF: Projétil de arma de fogo. PAS: Pressão arterial sistólica. a particularidades da extremidade traumatizada (perna distal e/ou pé) que, por suas características anatômicas, não permitiram um reparo vascular adequado.

É imperioso considerar o máximo de fatores na decisão de salvar ou não um membro mutilado, haja vista que tentativas prolongadas de salvamento de extremidade com lesão grave e complexa podem ser prejudiciais aos doentes, principalmente se todos estes esforços terminarem na amputação ${ }^{4}$.

Lesões combinadas podem inferir no prognóstico de salvamento de membro, entre elas, podemos citar: fraturas múltiplas, fratura Gustilo IIIC, transecção do nervo tibial ou ciático, isquemia prolongada (>6-12 h), lesão arterial infrapatelar, ligadura venosa, perda extensa de partes moles, cobertura inadequada dos tecidos moles do reparo vascular, contaminação severa da ferida, choque hipovolêmico com lesões associadas que ameaçam a vida, esmagamento, idosos com comorbidades, retardo no diagnóstico, retardo na cirurgia e fasciotomia tardia ${ }^{2,4,5}$.

Nestes casos, serviços norte-americanos têm demonstrado mais benefícios em relação a custos tanto hospitalares quanto em reabilitação e tempo de afastamento do trabalho, além de menores taxas de complicações, infecções e, muitas vezes, óbitos com uma postura agressiva como a amputação inicial ou primária corretamente indicada ${ }^{29-31}$, contudo, nos Estados Unidos, os pacientes têm melhores condições socioeconômicas para acesso a tecnologias avançadas no quesito de próteses para membros residuais, ao contrário do Brasil, onde a maioria dos usuários do Sistema Único de Saúde (SUS) tem acesso restrito a próteses e reabilitação, que, em alguns casos, tem sua qualidade insatisfatória.

Bondurant et al. $^{30}$, em uma revisão de 53 extremidades inferiores mutiladas, compararam amputação primária e tardia em relação à morbidade e ao custo. Os submetidos à amputação primária ficaram internados por cerca de metade do tempo com um custo cerca de $60 \%$ dos que foram submetidos à amputação tardia. Em relação à morbidade, nenhum doente do grupo amputação primária apresentou sepse ou óbito, contra seis casos no grupo de amputação tardia.

O reparo vascular com restauração bem sucedida da circulação, não determina necessariamente o salvamento do membro, tampouco a recuperação funcional, fatores que devem ser considerados numa situação de decidir qual a melhor abordagem terapêutica no trauma vascular de membros severamente agravados ${ }^{4}$.

Cabe apontar que a presente amostra também sofre um viés de seleção, por constituir amostragem de conveniência, uma vez que se trata de um serviço terciário 
que agrega casos graves e/ou que, muitas vezes, chegam à instituição hospitalar excedendo o tempo-limite para uma revascularização salvadora de membros. Este viés também ocorreu porque na região de abrangência deste hospital, com atendimento a uma população de aproximadamente dois milhões de habitantes, é o único hospital que realiza procedimentos de alta complexidade e que conta com equipes plantonistas tanto do serviço de cirurgia vascular quanto de ortopedista, cirurgião geral, cirurgião plástico e neurocirurgião. Não há, num raio de $400 \mathrm{~km}$, serviço/ hospital, com plantonistas de todas essas áreas juntas. O serviço de regulação de vagas Estadual e Municipal direciona os casos de trauma complexo e vascular apenas para o hospital em questão.

Este estudo permitiu concluir que a incidência de amputações primárias de membros no Hospital Santa Casa de Campo Grande-MS, foi maior em pacientes adultos jovens do sexo masculino e teve como principal causa lesões graves associadas decorrentes de acidente auto mobilístico.

São necessários, porém, novos estudos que enfoquem a prevalência desses traumas e consequentes procedimentos e comparem medidas profiláticas e melhorias nos treinamentos das equipes que atendem a traumas com redução do número de amputações.

Assim, espera-se que se obtenha maior solidez nas medidas redutoras desses indesejáveis índices.

\section{Referências}

1. De Luccia N. Amputação e reconstrução nas doenças vasculares e no pé diabético. Rio de Janeiro: Revinter; 2006. 248 p.

2. Araújo GM, Mathias SB, Felipe Junior G. Dados epidemiológicos. In: Murilo R, Brito C), Vergara E, Meirelles S. Trauma vascular. Rio de Janeiro: Revinter; 2006. p. 74-82.

3. De Luccia N. Amputações e a doença vascular periférica. J Vasc Br. 2004;3(3):179-80.

4. Campos-Christo SF, Miguel EV, Costa-Val R, Teixeira ELC. Amputação primária no trauma: indicações e aspectos legais. In: Murilo R, Brito C), Vergara E, Meirelles S. Trauma vascular. Rio de Janeiro: Revinter; 2006. p. 153-60.

5. Gregory RT, Gould RJ, Peclet $M$, et al. The mangled extremity syndrome index (MESI): a severity grading system for multisystem injury of the extremity. J Trauma. 1985;25:1147-50. http://dx.doi. org/10.1097/00005373-198512000-00005

6. Carmona GA, Hoffmeyer P, Herrmann FR, et al. Major lower limb amputations in the elderly observed over ten years: the role of diabetes and peripheral arterial disease. Diabetes Metab. 2005;31:449-54. http://dx.doi.org/10.1016/S12623636(07)70215-X

7. Seidel AC, Nagata AK, Almeida HC, Bonomo M. Epistemologia sobre amputações e desbridamentos de membros inferiores realizados no Hospital Universitário de Maringá. J vasc bras. 2008;7:308-15.
8. Santos CAS, Nascimento PFT. Desbridamento e amputações. In: Pitta GBB, Castro AA, Burihan E, editores. Angiologia e cirurgia vascular: guia ilustrado. Maceió: UNCISAL/ECMAL \& LAVA; 2003. http://www.lava.med.br/livro.

9. Regensteiner JG, Hiatt WR. Current medical therapies for patients with peripheral arterial disease: a critical review. Am J Med. 2002;112:49-57. http://dx.doi.org/10.1016/S00029343(01)01034-8

10. Shalabi R, Al Amri Y, Khoujah E. Vascular injuries of upper extremity. J Vasc Bras. 2006;5:271-6. http://dx.doi.org/10.1590/ S1677-54492006000400006

11. American Diabetes Association - ADA. Atualização: padrões e recomendações, patologias associadas. Diabetes Clin. 2000;4:118-36.

12. American Diabetes Association - ADA. Consensus development conference on diabetic foot wound care. Diabetes Care. 1999;22:1354-60. http://dx.doi.org/10.2337/diacare.22.8.1354

13. Schaan BD, Mandelli NCB. Conduta na doença arterial periférica em pacientes diabéticos. Rev Soc Cardiol RGS. 2004. http:// sociedades.cardiol.br/sbc-rs/revista/2004/02/artigo02.pdf.

14. Gamba MA, Gotlieb SLD, Bergamaschi DP, Vianna LAC. Amputações de extremidades inferiores por diabetes mellitus: estudo caso-controle. Rev Saude Publica. 2004;38:399-404. http:// dx.doi.org/10.1590/S0034-89102004000300010

15. Bild DE, Selby JV, Sinnock P, et al. Lower extremity amputation in people with diabetes: epidemiology and prevention. Diabetes Care. 1989;12:24. http://dx.doi.org/10.2337/diacare.12.1.24

16. Karlstrom L, Bergqvist D. Effects of vascular surgery on amputation rates and mortality. Eur J Vasc Endovasc Surg. 1997;14:273-83. http://dx.doi.org/10.1016/S1078-5884(97)80239-0

17. London. Department of Health and Social Security - DHSS. Amputation Statistics for England, Wales and Northern Ireland. London: DHSS; 1986.

18. Norgren L, Hiatt WR, Dormandy JA, et al. Inter-Society Consensus for the Management of Peripheral Arterial Disease (TASC II). J Vasc Surg. 2007;45(Suppl S):S5. http://dx.doi.org/10.1016/j. jvs.2006.12.037

19. Sheahan MG, Hamdan AD, Veraldi JR, et al. Lower extremity minor amputations: the roles of diabetes mellitus and timing of revascularization. J Vasc Surg. 2005;42:476-80. http://dx.doi. org/10.1016/j.jvs.2005.05.003

20. Assis MMA, Cerqueira EM, Nascimento MAA, Santos AM, Jesus WLA. Atenção primária à saúde e sua articulação com a estratégia saúde da família: construção política, metodológica e prática. Rev APS. 2007;10:189-99. http://www.nates.uff.br/novo/revista/ v010n2.htm.

21. Almeida Filho N, Rouquayrol MZ. Estudo de casos. In: Almeida Filho N, Rouquayrol MZ. Introdução a epidemiologia. 3. ed. Rio de Janeiro: Medsi; 2002. p. 196-205, 215-31.

22. De Godoy JM, De Godoy MF, Batigalia F, Trávolo AR, Monteiro EH. Lower-extremity amputation: a 6-year follow-up study in Brazil. J Orthop Surg (Hong Kong). 2005;13:164-6.

23. Diogo MJD. A dinâmica dependência-autonomia em idosos submetidos à amputação de membros inferiores. Rev Latino-Am Enfer. 1997;5:59-64. 
24. Sequeira FM, Martins AB. [Limb amputations carried out in hospitals of the national health service in the years from 1990 to 1993]. Acta Med Port. 1996;9(9-7):207-10.

25. Bergamini R. Um estudo sobre a população brasileira no século XX. Rev Eletr Desempr Zero. 2000. http://www.desempregozero. org.br/artigos/um_estudo_sobre_a_populacao_brasileira_no_ seculo_xx_fonte_ibge.php.

26. Meirelles SSL. Traumatismo arterial de membros inferiores. In: Pitta GBB, Castro AA, Burihan E, editores. Angiologia e cirurgia vascular: guia ilustrado. Maceió: UNCISAL/ECMAL \& LAVA; 2003. http:// www.lava.med.br/livro.

27. Belangero WD, Livani B, Angelini AJ, Davitt M. Amputaçäo dos membros inferiores na criança. Relato e experiência em 21 casos. Acta Bras Ortop. 2001;9:6-10.

28. Razuk A, Porciúncula M, Caffaro RA. Esmagamento de membros. In: Murilo R, Brito C), Vergara E, Meirelles S. Trauma vascular. Rio de Janeiro: Revinter; 2006. p. 423-33.

29. Durhan RM, Mistry BM, Mazuski JE, Shapiro M, Jacobs D. Outcome and utilityof scoring systems in the management of the mangled extremity. Am Surg 1996. 172:560-74.
30. Bondurant F), Cotler HB, Buckle R, Miller-Crotchett P, Browner $\mathrm{BD}$. The medical and economic impact of severely injured lower extremities. J Trauma. 1988;28:1270. http://dx.doi. org/10.1097/00005373-198808000-00023

31. Johasen $K$, Daines $M$, Helfet D. Objective criteria accurately predict amputation following lower extremity trauma. J Trauma. 1990;30:568-72. http://dx.doi.org/10.1097/00005373199005000-00007

Correspondência
Flavio Renato de Almeida Senefonte
Rua Antonio Marques Rodrigues, 20 - Mata do Jacinto
CEP 79033-291 - Campo grande (MS), Brasil
E-mail: flaviosenefonte@terra.com.br
Contribuições dos autores
Concepção e desenho do estudo: FRAS, GRPSR, MLC e MRC
Análise e interpretação dos dados: FRAS, GRPSR, MLC e ENN
Coleta de dados: TODOS OS AUTORES
Redação do artigo: FRAS, GRPSR, MLC e ENN
Revisão crítica do texto: TODOS OS AUTORES
Aprovação final do artigo*: TODOS OS AUTORES
Análise estatística: FRAS,GRPSR e ENN.
*Todos os autores leram e aprovaram a versão final submetida do J VasC Bras.
Responsabilidade geral pelo estudo TOSOS OS

